\title{
Simultaneous multi-telescope observations of FRB 121102
}

Document Version

Accepted author manuscript

Link to publication record in Manchester Research Explorer

\section{Citation for published version (APA):}

Caleb, M., Stappers, B., Rajwade, K., Driessen, L. N., Morello, V., Jankowski, F., Bezuidenhout, M. C., Malenta, M., \& Sanidas, S. (Accepted/In press). Simultaneous multi-telescope observations of FRB 121102. Royal Astronomical Society. Monthly Notices.

\section{Published in:}

Royal Astronomical Society. Monthly Notices

\section{Citing this paper}

Please note that where the full-text provided on Manchester Research Explorer is the Author Accepted Manuscript or Proof version this may differ from the final Published version. If citing, it is advised that you check and use the publisher's definitive version.

\section{General rights}

Copyright and moral rights for the publications made accessible in the Research Explorer are retained by the authors and/or other copyright owners and it is a condition of accessing publications that users recognise and abide by the legal requirements associated with these rights.

\section{Takedown policy}

If you believe that this document breaches copyright please refer to the University of Manchester's Takedown Procedures [http://man.ac.uk/04Y6Bo] or contact uml.scholarlycommunications@manchester.ac.uk providing relevant details, so we can investigate your claim.

\section{OPEN ACCESS}




\title{
Simultaneous multi-telescope observations of FRB 121102
}

\author{
M. Caleb, ${ }^{1 \star}$ B. W. Stappers ${ }^{1}$, T. D. Abbott $^{3}$, E. D. Barr ${ }^{2}$, M. C. Bezuidenhout ${ }^{1}$, \\ S. J. Buchner ${ }^{3}$, M. Burgay ${ }^{4}$, W. Chen ${ }^{2}$, I. Cognard ${ }^{5,6}$, L. N. Driessen ${ }^{1}$, R. Fender ${ }^{7}$, \\ G. H. Hilmarsson ${ }^{2}$, J. Hoang ${ }^{8}$, D. M. Horn ${ }^{3}$, F. Jankowski ${ }^{1}$, M. Kramer ${ }^{2}$, \\ D. R. Lorimer ${ }^{9}$, M. Malenta ${ }^{1}$, V. Morello ${ }^{1}$, M. Pilia ${ }^{4}$, A. Possenti ${ }^{10}$, K. M. Rajwade ${ }^{1}$, \\ A. Ridolfi ${ }^{4,2}$, L. Rhodes ${ }^{7,2}$, S. Sanidas ${ }^{1}$, M. Serylak ${ }^{3,11}$, L. G. Spitler ${ }^{2}$, \\ L. J. Townsend ${ }^{12,3}$, A. Weltman ${ }^{13}$, P. A. Woudt ${ }^{12}$, J. Wu ${ }^{2}$ \\ ${ }^{1}$ Jodrell Bank Centre for Astrophysics, Department of Physics and Astronomy, The University of Manchester, Manchester, M13 9PL, UK \\ ${ }^{2}$ Max-Planck-Institut für Radioastronomie, Auf dem Hügel 69, D-53121 Bonn, Germany \\ ${ }^{3}$ South African Radio Astronomy Observatory, 2 Fir Street, Black River Park, Observatory 7925, South Africa \\ ${ }^{4}$ INAF - Osservatorio Astronomico di Cagliari, via della Scienza 5, I-09047 Selargius (CA), Italy \\ ${ }^{5}$ Laboratoire de Physique et Chimie de l'Environnement et de l'Espace, Université d'Orléans/CNRS, F-45071 Orléans Cedex 02, France \\ ${ }^{6}$ Station de radioastronomie de Nançay, Observatoire de Paris, CNRS/INSU, F-18330 Nançay, France \\ ${ }^{7}$ Astrophysics, Department of Physics, University of Oxford, Keble Road, Oxford OX1 3RH, UK \\ ${ }^{8}$ Instituto de Particulas y Cosmologia (IPARCOS), Universidad Complutense de Madrid, Madrid, Spain \\ ${ }^{9}$ Department of Physics and Astronomy, West Virginia University, PO Box 6315, Morgantown, WV 26506, USA \\ ${ }^{10}$ Università degli Studi di Cagliari, Dip. Fisica, S.P. Monserrato-Sestu Km 0.700, I-09042 Monserrato (CA), Italy \\ ${ }^{11}$ Department of Physics and Astronomy, University of the Western Cape, Bellville, Cape Town 7535, South Africa \\ ${ }^{12}$ Department of Astronomy, University of Cape Town, Private Bag X3, Rondebosch 7701, South Africa \\ ${ }^{13}$ High Energy Physics, Cosmology \& Astrophysics Theory (HEPCAT) group, Department of Mathematics and Applied Mathematics, \\ University of Cape Town, 7701 Rondebosch, Cape Town, South Africa,
}

\begin{abstract}
We present 11 detections of FRB 121102 in 3 hours of observations during its 'active' period on the 10th of September 2019. The detections were made using the newly deployed MeerTRAP system and single pulse detection pipeline at the MeerKAT radio telescope in South Africa. Fortuitously, the Nançay radio telescope observations on this day overlapped with the last hour of MeerKAT observations and resulted in 4 simultaneous detections. The observations with MeerKAT's wide band receiver which extends down to relatively low frequencies $(900-1670$ $\mathrm{MHz}$ usable L-band range) have allowed us to get a detailed look at the complex frequency structure, intensity variations and frequency-dependent sub-pulse drifting. The drift rates we measure for the full-band and sub-banded data are consistent with those published between $600-6500 \mathrm{MHz}$ with a slope of $-0.15 \pm 0.01$. Two of the detected bursts exhibit fainter 'precursors' separated from the brighter main pulse by $\sim 28 \mathrm{~ms}$ and $\sim 34 \mathrm{~ms}$, suggestive of compact emission regions in the progenitor. A follow-up multi-telescope campaign on the 6th and 8th October 2019 to better understand these frequency drifts and structures over a wide and continuous band was undertaken. No detections resulted, indicating that the source was 'inactive' over a broad frequency range during this time.
\end{abstract}

Key words: instrumentation: interferometers - intergalactic medium - surveys - radio continuum: transients - methods: data analysis

\section{INTRODUCTION}

Fast radio bursts (FRBs) are characterised by millisecond-duration, $\sim$ Jy-level, bright radio pulses appearing at random locations in the

^ E-mail: manisha.caleb@manchester.ac.uk sky and originating at cosmological distances (e.g. Thornton et al. 
2013). However, the nature of the sources producing these FRBs is not known. Their large dispersion measures (DMs) which are the integrated electron column densities along the lines-of-sight, are believed to be effective proxies for distance (e.g. Lorimer et al. 2007; Thornton et al. 2013). The measured DM values significantly exceed the maximum Galactic contribution (see Petroff et al. 2016, for details) from the Milky Way's interstellar medium (ISM) implying distances of several gigaparsecs. The combination of cosmological origin of these pulses, along with their estimated high radio luminosities and correspondingly large brightness temperatures is what makes FRBs compelling. Almost every radio telescope in the world is currently undertaking large-area surveys at radio frequencies ranging from $100 \mathrm{MHz}$ (Coenen et al. 2014; Karastergiou et al. 2015; Tingay et al. 2015) up to tens of GHz (Shannon et al. 2018; Michilli et al. 2018; Gajjar et al. 2018) to discover, study and understand these bursts.

The FRBs discovered to date show a remarkable diversity of observed properties in terms of spectral and temporal variations, polarization properties and repeatability. Perhaps, the most striking difference is the repeatability. Hundreds of hours of telescope time have been spent on the follow-up observations of known FRBs (e.g. Rane \& Lorimer 2017), yet only some of them have been observed to repeat (Spitler et al. 2016; Shannon et al. 2018; The CHIME/FRB Collaboration et al. 2019) for a given sensitivity limit. Presently, instrumental sensitivity and time spent following up a known FRB field to look for repeats, are the two major reasons for the observed dichotomy (Caleb et al. 2018; Kumar et al. 2019) if all FRBs are indeed repeating sources.

The nature of FRBs, and that of the repeating FRBs in particular, may be revealed from their emission properties. Hence, understanding the flux density spectra is of great importance. The first repeating FRB 121102 (Spitler et al. 2014a) has been localised to a low-metallicity, low-mass dwarf galaxy at $z \sim 0.2$ (Tendulkar et al. 2017), and studied extensively across multiple wavelengths and frequencies. Until now, most successful observations of this FRB have been carried out above $1 \mathrm{GHz}$ (Gourdji et al. 2019), with bursts detected at frequencies as high as $8 \mathrm{GHz}$ (e.g. Gajjar et al. 2018). More recently, a single burst from this FRB was reported in the upper half of the CHIME $400-800$ MHz band (Josephy et al. 2019).

The spectral behaviour of FRBs seen so far is very unusual. This is especially true for FRB 121102, where the bursts are dominated by patches of bright emission with varying spectral indices across the bands. Though Galactic pulsars and magnetars are also seen to exhibit complex structures in the time domain, FRB 121102's pulses are strikingly different due to their enormous energies. High-time resolution $(\sim 10 \mu \mathrm{s})$ observations of FRB 121102 with the Arecibo telescope in the $1.1-1.7 \mathrm{GHz}$ band show bursts with complex time-frequency structures and sub-bursts (Hessels et al. 2019). The sub-bursts are seen to drift towards lower frequencies at later times within the burst envelope by $\sim 200 \mathrm{MHz} \mathrm{ms}^{-1}$ in the $1.1-1.7 \mathrm{GHz}$ band. The shortest separation between two distinctive bursts so far reported is $\sim 26$ $\mathrm{ms}$ (Gourdji et al. 2019). This separation may represent an upper limit on a possible underlying periodicity, or these pulses may have come from a single emission window that is a small fraction of a possible period (Hardy et al. 2017).

Continued long term monitoring of FRB 121102 is ongoing at various facilities around the world, to understand its nature and burst properties. It is evident from the literature over the years and these campaigns, that FRB 121102 exhibits sudden periods of activity with no statistically significant periodicity determined, followed by long periods of quiescence (Oppermann et al. 2015; Gourdji et al. 2019; Zhang et al. 2018). The Five-hundred-metre Aperture Spherical Telescope (FAST) in China reported several tens of detections of FRB 121102 pulses using their L-band (1.1 $1.5 \mathrm{GHz}$ ) array of 19-beams, between 29th and 31st August inclusive ( $\mathrm{Di}$ et al. 2019). Consequently, we carried out observations of FRB 121102 during this 'active' period as part of a Director's Discretionary Time (DDT) proposal at the MeerKAT radio telescope.

In this paper we present our observations and detections of FRB 121102 with MeerKAT, which has allowed us to get a detailed look at a possible periodicity in the pulses due to the presence of 'precursor' bursts, the complex frequency structure, and also the sub-pulse frequency drifting of some bursts. In Section 2 we present the observational configuration of the MeerKAT telescope, and our burst detections and data anaylsis. In Section 3, we present the subsequent multi-telescope campaign organised to study possible FRB 121102 bursts over a wide and continuous frequency band to study pulse spectral and temporal evolution. The results from our detections/non-detections are presented and discussed in Section 4, following which we present our conclusions in Section 5.

\section{THE MEERKAT RADIO TELESCOPE: OBSERVATIONS AND DATA ANALYSES}

The South African Radio Astronomy Observatory (SARAO) Meer(more) Karoo Array Telescope (MeerKAT; Jonas \& MeerKAT Team 2016; Camilo et al. 2018; Mauch et al. 2020) is a radio interferometer consisting of 64, $13.96 \mathrm{~m}$ dishes in the Karoo region in South Africa. The dishes are spread out over $8 \mathrm{~km}$ with 40 dishes in the inner $\sim 1 \mathrm{~km}$ core. In these observations, MeerKAT is operating at a centre frequency of $1284 \mathrm{MHz}$ and a usable bandwidth of $~ 770 \mathrm{MHz}$. The Meer(more) TRAnsients and Pulsars (MeerTRAP) project at the MeerKAT telescope undertakes fully commensal, high time resolution searches of the transient radio sky, simultaneously with all the other ongoing MeerKAT Large Survey Projects. Using the MeerTRAP backend (see Section 2.1 for details), MeerKAT observes simultaneously in two modes: incoherent and coherent. In the coherent mode, the data from the inner 40 dishes in the $\sim 1 \mathrm{~km}$ core of the MeerKAT array, are coherently combined to form a number of beams on sky with a sensitive Field-of-View (FoV). Typically, we form 396 beams on sky with a combined FoV of $\sim 0.2 \mathrm{deg}^{2}$. In the incoherent mode the intensities of all 64 MeerKAT dishes are added to create a less sensitive but much wider FoV of $\sim 1 \mathrm{deg}^{2}$.

The first DDT observations were carried out on the 10th of September 2019 starting at 03:43 UT for a duration of 3 hours. The observations were performed with the MeerTRAP backend system, which utilises state-of-the-art Graphics Processing Units (GPUs) allowing real time searches of the tied-array beams formed on the sky. For these observations, the well constrained position of FRB 121102 allowed us to use a phased array of 60 MeerKAT dishes. MeerKAT uses an FX correlator (Camilo et al. 2018) and the MeerTRAP transient search pipeline (see Section 2.1 for details) uses the F-engine output stream for ingest. The F-engine is configured to channelize the $856 \mathrm{MHz}$ wide band into 4096 channels with a native time resolution of $4.785 \mu \mathrm{s}$. The geometric delays as well as those determined from observations of a strong calibrator J0408-6545 are applied to the data stream in the telescope F-engine, thus phasing up the array. The complex voltage data are channelized and then sent over the Central 
BeamFormer (CBF) network to the beamforming User Supplied Equipment (FBFUSE) that was designed and developed at the Max-Planck-Institut für Radioastronomie in Bonn. FBFUSE combines this data from the dishes into the requested number of total intensity tied-array beams which are placed at the desired locations within the primary beam of the array. FBFUSE also combines time samples, to give an effective sampling rate of approximately $306.24 \mu \mathrm{s}$. The beams are then put back onto the network where they are captured by the Transient User Supplied Equipment (TUSE), a real-time transient detection instrument developed by the MeerTRAP ${ }^{1}$ team at the University of Manchester.

\subsection{Transient detection instrument}

TUSE consists of 67 Lenovo servers with one head node and 66 compute nodes. Each compute node contains two Intel Xeon CPU processors, each possessing 16 logical cores for computation, two Nvidia GeForce 1080 Ti Graphical processing units (GPUs) and 256 GB of DDR4 Random Access Memory (RAM) blocks. Each of the nodes is connected to a breakout switch via $10 \mathrm{GbE}$ network interface cards (NIC) that are used to ingest data coming from FBFUSE. Data from FBFUSE are received over the network on the NICs as SPEAD2 ${ }^{2}$ packets that are read by the data ingest code and written to POSIX shared memory ring buffers of 50 seconds duration. The data are arranged such that each compute node processes a number of coherent beams to be processed in real-time. Since the data from the beamformer come in a frequency-time format (i.e. frequency being the slowest axis), they are transposed to a time-frequency format on a per beam basis, that are required by the search code. The resulting filterbank data are saved in separate shared memory buffers corresponding to each beam. More details on TUSE will be presented in an upcoming paper (Stappers et al. in prep).

For this targeted observation, since the position of FRB 121102 is well-known to within a few milliarcseconds, we were able to run in a mode where only 2 nodes with one beam per node were processing the data in real time. Only extracted candidates were saved for further examination.

\subsection{Single pulse search pipeline}

The data for each beam are searched for bright bursts using the state-of-the-art, GPU-based single pulse search pipeline AstroAccelerate $^{3}$ (Dimoudi \& Armour 2015; Adámek \& Armour 2016; Adámek et al. 2017; Dimoudi et al. 2018; Adámek \& Armour 2019). The real-time search was done by incoherently de-dispersing in the DM range $0-5118.4 \mathrm{pc} \mathrm{cm}^{-3}$, divided into multiple sub-ranges with varying DM steps and time averaging factors. We also searched up to a maximum boxcar width of $0.67 \mathrm{~s}$. For the region containing the DM of the FRB 121102, $380.68-771.88 \mathrm{pc} \mathrm{cm}^{-3}$, the DM step and the effective sampling time were $0.652 \mathrm{pc} \mathrm{cm}^{-3}$ and $612.8 \mu$ s respectively. This particular choice of parameters allowed us to process all the data in real time, thanks to strict optimisations applied in the AstroAccelerate algorithms.

To reduce the number of detections due to Radio Frequency

\footnotetext{
1 https://www.meertrap.org/

2 https://casper.ssl.berkeley.edu/wiki/SPEAD

3 https://github.com/AstroAccelerateOrg/astro-accelerate
}

Interference (RFI), we applied a static frequency channel mask to the data before the de-dispersion and single-pulse search. The RFI remained stable throughout the observations, meaning that our choice of static mask was sufficient. Additionally, the data were cleaned using standard zero-DM excision (Eatough et al. 2009) to remove any remaining broadband RFI that was infrequent enough not to be included in the mask. The extracted candidate files contained raw filterbank data of the dispersed pulse and additional padding of $0.5 \mathrm{~s}$ at the start and at the end of the file.

\subsection{MeerKAT detections}

The DDT observations on 10th September 2019 resulted in the detection of a total of 11 pulses from FRB 121102 in $~ 3$ hours of observations. The pulse profiles are shown in Figure 1 and their properties are shown in Table 1. From Figure 1 it is evident that bursts 07,10 and 11 exhibit sub-structure. The analysis of the subbursts and the structure-optimized DM will be reported in Platts et al. (in prep). We note that bursts 02, 06 and 09 are narrower in frequency and less likely to be affected by drifting substructure. As a result, bursts 02,06 and 09 have been de-dispersed to the signalto-noise (S/N) maximising DMs of 566.1, 563.2 and 565.2 $\mathrm{pc} \mathrm{cm}^{-3}$ respectively. For this work, all other bursts have been de-dispersed to the average DM of bursts 02,06 and 09 (i.e. $564.8 \mathrm{pc} \mathrm{cm}^{-3}$ ) in Figure 1 . The time series for each de-dispersed pulse was convolved with a series of Gaussian template profiles over a range of widths using the python based package $\operatorname{SPYDEN}^{4}$ to obtain the width and the best fit $\mathrm{S} / \mathrm{N}$ for each pulse reported in Table 1 .

Incidentally, the Nançay radio telescope (NRT) was observing FRB 121102 on the same day and their observations overlapped with the last hour of MeerKAT observations. This resulted in the simultaneous detections of 4 bursts (Bursts 08, 09, 10 and 11 in Figure 2), the analyses and details of which are discussed in Section 2.4. Two of the MeerKAT detections (Bursts 03 and 05 in Figure 2) show fainter 'precursors' separated from the main pulse by $\sim 28$ $\mathrm{ms}$ and $\sim 34 \mathrm{~ms}$ respectively with the signal level between the main burst and the precursor equal to the noise floor. We analysed our MeerKAT beam-formed data for the 11 pulses in great detail, and observed the spectra to vary across frequency as a function of time for most bursts. The MeerKAT detections reveal both narrow-band bursts and some that exhibit complex time-frequency structures and drifts. Similar spectral variation in bursts has also been reported by previous studies (Spitler et al. 2014b; Michilli et al. 2018; Hessels et al. 2019) but never over such a large frequency range near 1.3 GHz.

The sensitivity and large bandwidth of MeerKAT has resulted in a series of intriguing bursts which show structure and intensity variations across the entire band, as in Figure 1. We are able to probe down to the lower frequencies (rarely studied in the published bursts), and in most bursts there seems to be an interesting change at frequencies around $1200 \mathrm{MHz}$. At these frequencies, the source either becomes significantly fainter or we see complex bifurcated structure. It has been noted that in higher time resolution ( $\sim 10 \mu \mathrm{s})$ observations, there is a tendency for the central frequency of a bandlimited sub-burst to drift to lower frequencies at later times during the burst (Hessels et al. 2019). It has also been suggested that radio pulses can be strongly amplified by lensing in local ionised material (Main et al. 2018). This adds support to the proposal that the bursts from FRB 121102 might be lensed by host galaxy plasma (Hessels

\footnotetext{
${ }^{4}$ https://bitbucket.org/vmorello/spyden/src/master/
} 
Table 1. Observed properties of bursts detected with MeerKAT. We note that the $\mathrm{S} / \mathrm{N}$-optimised DM causes potential sub-bursts to overlap in time and sweep upward in frequency. As a result we do not quote DMs. Updated structure-optimized DMs and their errors will appear in Platts et al. (in prep). See Section 2.3 for details on DM.

\begin{tabular}{lcccc}
\hline \hline Burst & $\begin{array}{c}\text { Arrival time } \\
\text { (Barycentric MJD) }\end{array}$ & S/N & $\begin{array}{c}\text { Width } \\
(\mathrm{ms})\end{array}$ & $\begin{array}{c}\text { Waittime } \\
(\mathrm{s})\end{array}$ \\
\hline 01 & 58736.148545185752 & 12.2 & 6.3 & $\ldots$ \\
02 & 58736.165647624010 & 97.7 & 2.0 & 1477.65 \\
03 & 58736.165664566521 & 58.4 & 3.7 & 1.46 \\
04 & 58736.166942733849 & 15.5 & 12.0 & 110.43 \\
05 & 58736.184839564114 & 108.0 & 2.1 & 1546.28 \\
06 & 58736.200201955864 & 39.0 & 2.5 & 1327.31 \\
07 & 58736.211554436508 & 112.8 & 10.7 & 980.85 \\
08 & 58736.235176574322 & 88.7 & 2.6 & 2040.95 \\
09 & 58736.240623411504 & 45.6 & 2.3 & 470.60 \\
10 & 58736.247856676535 & 32.7 & 4.2 & 624.95 \\
11 & 58736.254215477995 & 208.8 & 10.7 & 549.40 \\
\hline
\end{tabular}

et al. 2019) thereby leading to the observed variable magnification, narrow frequency structures, and clustered arrival times of highly amplified pulses.

\subsection{Simultaneous detections with MeerKAT and the NRT}

Bursts 08, 09, 10 and 11 were detected simultaneously with the MeerKAT and the NRT on the 10th September of 2019 and are shown in Figure 2. The burst structure observed in the $1200-1700$ $\mathrm{MHz}$ band of MeerKAT is similar to the frequency characteristics we see in the corresponding NRT band. NRT's higher time resolution of $64 \mu$ s compared to MeerTRAP's $306.24 \mu$ s reveals sharp detailed structure in the high frequency half of the band, while MeerKAT's better sensitivity and larger bandwidth allow us to see more structure across the band. For reference, the intrachannel DM smearing times for $565 \mathrm{pc} \mathrm{cm}^{3}$ are $\sim 463 \mu \mathrm{s}$ and $\sim 854 \mu$ s at the MeerKAT and NRT frequencies of $1284 \mathrm{MHz}$ and $1400 \mathrm{MHz}$ respectively. Applying the radiometer equation with a system equivalent flux density (SEFD) of $25 \mathrm{Jy}$ to flux calibrate the NRT data leads to peak fluxes of $0.61,0.29,0.17$ and $1.06 \mathrm{Jy}$ and widths of 3.2, 3.3, 4.0 and $5.1 \mathrm{~ms}$ respectively for the four detected bursts with the NRT. The estimated flux densities and measured widths are comparable to the MeerKAT values listed in Table 1. These simultaneous detections help us verify and characterise the MeerTRAP transient detection system.

\section{MULTI-TELESCOPE CAMPAIGN}

Observing over the widest possible frequency range simultaneously would help us understand how the structure we see relates to the drifting features seen in other publications, and also whether there are multiple instances of these apparent changes in burst properties as a function of frequency. Multi-frequency radio observations across a wide and continuous spectral range would provide valuable insight and new information on the emission/propagation effects. To this end, following the successful detections of 11 pulses from FRB 121102 on 10th September 2019, we organised a joint observing campaign on the 6th and 8th of October 2019, between the MeerKAT $(900-1670 \mathrm{MHz})$, Nançay $(1100-1800 \mathrm{GHz})$ and Effelsberg $(4000-8000 \mathrm{GHz})$ radio telescopes to better understand these frequency drifts and structures over a wide and continuous band.

\subsection{The MeerKAT radio telescope}

On October 6th and 8th 2019, the data were recorded according to the telescope specifications in Section 2, and processed in real-time using the transient detection instrument and search pipeline detailed in Sections 2.1 and 2.2. In addition to the real-time processing in Section 2.1, two additional nodes were used to record the data to disk at a full data rate for additional offline processing. No bursts were detected above a $\mathrm{S} / \mathrm{N}$ of 10 by the real-time pipeline. The recorded data were processed offline for single pulses down to a lower $\mathrm{S} / \mathrm{N}$, as well as for a possible periodicity given the detections of 'precursors' from the data taken on the 10th of September 2019.

\subsubsection{Periodicity searches}

We incoherently de-dispersed the data recorded on the 6th and 8th of October 2019 over a range of trial DMs, $540.0 \leq \mathrm{DM} \leq$ $590.0 \mathrm{pc} \mathrm{cm}^{-3}$ in steps of $1 \mathrm{pc} \mathrm{cm}^{-3}$. For each trial DM, the resulting de-dispersed time series was searched for short period pulsations using the Fast Fourier Transform (FFT). The FFT search was performed on the recorded data using the PRESTO suite of pulsar search and analysis software (Ransom et al. 2002). The realfft routine was used to perform an FFT on each de-dispersed time series following which the accelsearch routine was used to sum 16 harmonics incoherently for each frequency bin to improve the S/N. All detections above a $\mathrm{S} / \mathrm{N}$ of 5 were saved for further inspection. We folded the time series for each candidate period using the prepfold routine and the folded time series was visually inspected to check whether it resembled a true astrophysical source. No significant periodic pulsations were detected above the threshold S/N.

A significant degree of sensitivity is lost during incoherent harmonic summing in FFT searches. Moreover, red-noise in the data can be an important factor when searching for longer periods. Therefore, we decided to perform a Fast Folding Algorithm search on the data using the RIPTIDE ${ }^{5}$ FFA algorthm developed by Morello et al. (in prep) to search for periods ranging from 500 milliseconds to 10 minutes. The advantage of the FFA is that since we performed the search in the time domain, we did not lose any sensitivity to harmonic summing and were equally sensitive to a large range of pulse periods. Similar to the FFT, we de-dispersed and folded each time series and vetted the candidates for significant pulse profiles above a $\mathrm{S} / \mathrm{N}$ of 8 . We did not detect any significant periodic pulsations above the $\mathrm{S} / \mathrm{N}$ threshold.

\subsubsection{Offline single pulse search}

We processed the recorded MeerTRAP data obtained on 2019 October 6 and 8 using the HEIMDALl single-pulse search pipeline ${ }^{6}$. We performed both a coarse blind search and a finer targeted search centred around FRB 121102's nominal DM. The coarse search was done for trial DMs $[0,4000] \mathrm{pc} \mathrm{cm}^{-3}$ with a 5 per cent $\mathrm{S} / \mathrm{N}$ loss tolerance and the fine search was performed between DMs $[0,700] \mathrm{pc} \mathrm{cm}^{-3}$ with a 1 per cent tolerance. The maximum boxcar filter width was 4096 samples, or about $313.6 \mathrm{~ms}$. The frequency channels that were known to be affected by RFI at that

\footnotetext{
5 https://bitbucket.org/vmorello/riptide/src/master/

6 https://sourceforge.net/projects/heimdall-astro/
} 

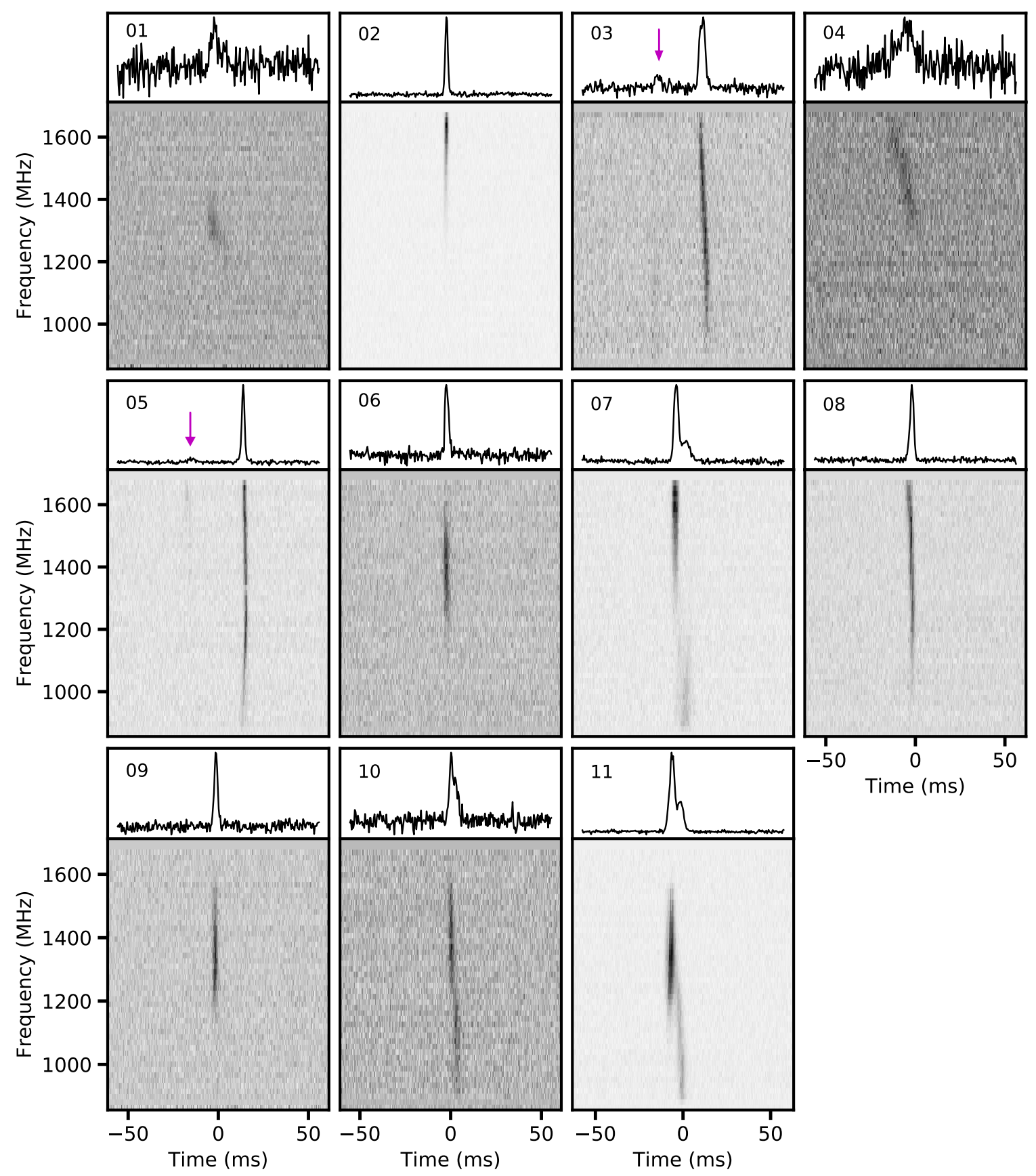

Figure 1. Dynamic spectra of the bursts detected with MeerKAT. The top panel in each pulse shows the frequency-averaged pulse profile. The bottom panel shows de-dispersed frequency spectrum in which the frequency resolution of the bursts has been decimated to 64 channels each in order to make them more visible. The time resolution of the pulses is $306.24 \mu \mathrm{s}$. Bursts 02, 06 and 09 have been de-dispersed to the S/N maximising DMs of 566.1, 563.2 and 565.2 pc $\mathrm{cm}^{-3}$ respectively. All other bursts have been de-dispersed to an average DM of $564.8 \mathrm{pc} \mathrm{cm}^{-3}$ (see Section 2.3 for details). We note that we did not utilize the standard RFI excision mask detailed in Section 2.2 and instead cleaned the data manually. The data are uncalibrated, and the flux densities are in arbitrary units. Bursts 03 and 05 are seen to show 'precursor' bursts whose positions are indicated by the downward arrows.

time were masked during the HEIMDALL run, leaving us with about $491 \mathrm{MHz}$ of usable bandwidth. Standard zero-DM RFI excision was performed. We extracted the resulting candidates using the DSPSR software package (van Straten \& Bailes 2011) and we visualised them using PSRCHIVE (Hotan et al. 2004) tools. We then visually inspected all candidates from both the coarse and fine search down to a $\mathrm{S} / \mathrm{N}$ threshold of 6 . No bursts were detected above the threshold.

\subsection{The Sardinia Radio Telescope}

The 64-m Sardinia Radio Telescope (SRT; Bolli et al. 2015) observed FRB 121102 on the 6th of October starting at UT 

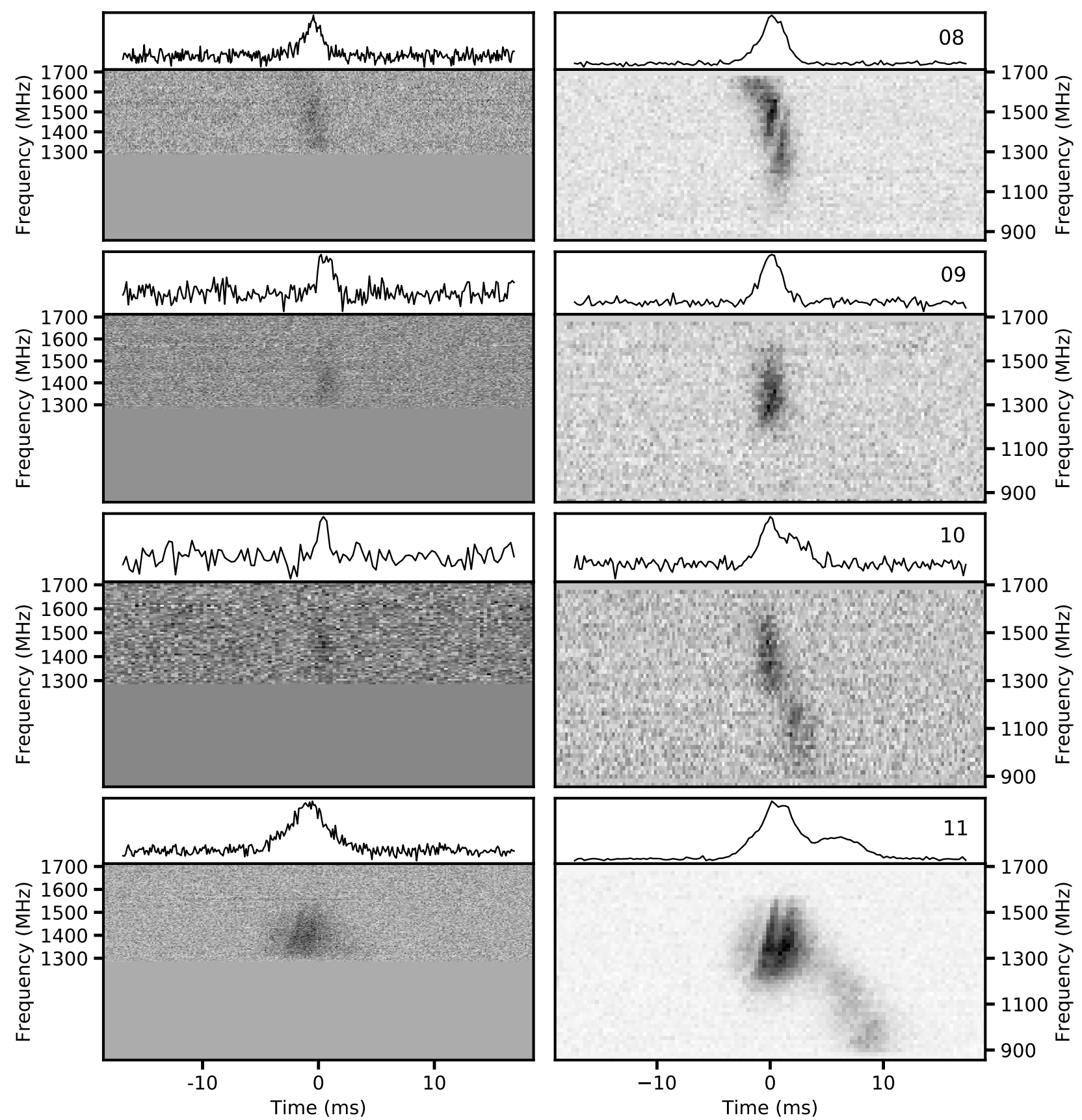

Figure 2. Dynamic spectra of bursts $08,09,10$ and 11 detected simultaneously with MeerKAT ( $856 \mathrm{MHz}$ bandwidth) and Nançay (512 MHz bandwidth) radio telescopes. The top panel in each pulse shows the frequency-averaged pulse profile. The pulses detected with Nançay are plotted in the left column and the MeerKAT pulses are shown in the right column. The Nançay and corresponding MeerKAT pulses in each row have been de-dispersed to the DMs in Section 2.3. The four MeerKAT pulses have 64 frequency channels each, with a time resolution of $306.24 \mu$ s. The Nançay pulses from top to bottom have $128,64,64$ and 128 channels, with time resolutions of $256 \mu \mathrm{s}, 128 \mu \mathrm{s}, 1.024 \mathrm{~ms}$ and $128 \mu \mathrm{s}$ respectively.

01:45:42 for a total of 195 minutes. Baseband data were acquired with the LEAP ROACH1 backend (Bassa et al. 2016) covering a total bandwidth of $80 \mathrm{MHz}$ centered at $336 \mathrm{MHz}$ (the P-band of SRT's coaxial L-P band receiver; Valente et al. 2010). To minimise packet loss, the observation was split into 30-minute long segments (except for the last one, with a duration of 15 minutes) with $\sim 1$ minute gaps.

The search for single pulses was done offline using both the
Presto and HeIMDALl software packages. The first step of the analysis was to convert the baseband data, recorded in $\mathrm{DADA}^{7}$ (Distributed Acquisition and Data Analysis) format into a filterbank format, compatible with both PRESTo and HeIMDALL. This was done using the digifil routine of the PSRCHIVE

\footnotetext{
7 http://psrdada.sourceforge.net/
} 
package, creating a total-intensity 320-channels file, coherently de-dispersed at the dispersion measure of FRB 121102, with a sampling time of $4 \mu \mathrm{s}$. The file was subsequently re-sampled with a final sampling time of $512 \mu \mathrm{s}$. For the analysis based on PrESTO, we first removed RFI using rfifind (preliminarily flagging the frequency channels with the most prominent known RFI). The time series were de-dispersed with $200 \mathrm{DM}$ steps covering the range 552-572 pc $\mathrm{cm}^{-3}$ and analysed with single_pulse_search.py using a minimum $\mathrm{S} / \mathrm{N}$ of 10 . The high threshold was adopted because of the possibility of RFI severely affecting the P-band due to the absence of the shielding cover usually installed on the Gregorian dome during low-frequency observations at the SRT. The analysis with HEIMDALL, also including a cross-match between candidates at adjacent DMs and times, was done on the same range of dispersion measures using a pulse width threshold of $2^{5}$ time bins of 16.384 $\mu \mathrm{s}$ and a minimum $\mathrm{S} / \mathrm{N}$ of 10 . No reliable pulse candidates were found by either method. Since the data were uncalibrated and the level of RFI severe, it is not possible to give a reliable flux density upper limit for our search.

\subsection{The Effelsberg Radio telescope}

We observed FRB 121102 with the Effelsberg-100 m radio telescope on 2019 October 6th and 8th for 240 and 360 minutes, respectively. The observations started at 04:25:00 UTC on the 6th and at 01:45:00 UTC on the 8th. No bursts were detected during our observations. We used the S45mm single pixel receiver, which observes at 4$8 \mathrm{GHz}$ and has an SEFD of $25 \mathrm{Jy}$ averaged across the band. The data were recorded with full Stokes using two ROACH2 backends, and were in a DADA format. The data have 4096 channels, each with a bandwidth of $0.976562 \mathrm{MHz}$, and a time resolution of 131 $\mu \mathrm{s}$.

To search for single pulses, we used the PREsto software package. In order to make our data compatible with Presto we extracted Stokes I from the data in a Sigproc filterbank format. By using rfifind we created an RFI mask to apply to the data. We then created de-dispersed time series between $0-1000 \mathrm{pc} \mathrm{cm}^{-3}$ in steps of $2 \mathrm{pc} \mathrm{cm}^{-3}$, in which we searched for single pulses using single_pulse_search.py with a $\mathrm{S} / \mathrm{N}$ threshold of 7 . The candidates were inspected by eye using waterfaller.py, which plots their dynamic spectra.

\subsection{Nançay Radio telescope}

The Nançay Radio Telescope (NRT) is a Kraus design meridian instrument equivalent to a 94-m dish. FRB 121102 was observed on the 10th of September and 6th of October 2019. On Sept 10th, the observation started at 05:29:40 UTC for 75 minutes while on Oct 6th, the observation started at 03:50:55UTC for 71 minutes. Due to technical issue, no observation was performed on Oct 8th. The Low Frequency receiver (1.1-1.8GHZ) was used and a total bandwidth of $512 \mathrm{MHz}$ was processed through the search mode of NUPPI (Nançay Ultimate Pulsar Processing Instrument; Desvignes et al. 2011). In this mode, 1024 channels of width $0.5 \mathrm{MHz}$ are recorded as 4-bit in total intensity samples every $64 \mu \mathrm{s}$. The search for single dispersed bursts was performed offline using the PREsto software. The data were cleaned with rfifind and de-dispersed with 128 $\mathrm{DM}$ values covering the range $527-591 \mathrm{pc} \mathrm{cm}^{-3}$ with $0.5 \mathrm{pc} \mathrm{cm}^{-3}$ steps. The PRESTO script single_pulse_search.py searched for individuals bursts using different averaging window widths. All candidates above $\mathrm{S} / \mathrm{N}$ of 6.5 were inspected by eye revealing 4 bursts shown in Figure 2 detected on the 10th of September, and none on the 6th of October.

\section{RESULTS AND DISCUSSION}

As evident from Figures 1 and 2 the MeerKAT pulses of FRB 121102 show intricate frequency structure similar to that reported by Hessels et al. (2019). The analysis of the sub-bursts and the structure-optimized DM is not the focus of this paper and will be reported in Platts et al. (in prep).

\subsection{Drift rate analysis}

The pulses in Figure 1 are seen to exhibit complex structure in frequency. Hessels et al. (2019) show that similar single peak pulses in their sample can be separated in distinct components called subbursts, when de-dispersed to the structure-optimized DM as opposed to the S/N-optimized DM. These subbursts are seen to exhibit a progressive drift towards lower frequencies at later times in the burst (e.g. Hessels et al. 2019). Here we estimate the drift rates that were observed in select pulses from the sample of 11 bursts in Figure 1. The method and analyses of the DMs which maximize structure in order to characterize sub-burst drifting will be presented in Platts et al. in prep. We note that the structure optimized DM is more representative of the true DM of those bursts which exhibit structure. Not all pulses in the sample were observed to exhibit drifting structure. Though most appear to be sharp pulses, they could be a result of our relatively coarse time resolution of $306.24 \mu \mathrm{s}$. However, many pulses from FRB 121102 are indeed sharp and do not exhibit downward drifting sub-structure.

The on-pulse regions of the pulses after de-dispersing to the structure-optimized DM (see Platts et al. in prep) were extracted and we computed a 2D Auto-Correlation Function (ACF),

$\operatorname{ACF}(\tau, v)=\int_{0}^{t} \int_{v}^{0} f(t, v) f\left(t-\tau, v-v^{\prime}\right) \mathrm{dt} \mathrm{d} v$,

where $v^{\prime}$ and $\tau$ are the frequency and time lags respectively. An example of the ACF analysis for burst 11 is shown in Figure 3. Assuming the pulse profile, spectral bandwidth of the burst envelope and subbursts are well described by a Gaussian, we measure their FWHMs from a Gaussian profile fit to the summed autocorrelation over the respective axes. A tilt in the autocorrelation ellipse reflects the drift rate in $\mathrm{MHz} \mathrm{ms}^{-1}$. Similar to Hessels et al. (2019), we note that there is a tendency for the sub-bursts to drift to lower frequencies at later times during the burst. We successfully measured the drifts using the ACF method for bursts 07 and 11. Given our large bandwidth, burst 07 was sub-banded and the drift rates were measured to be $33 \pm 0.03$ $\mathrm{MHz} / \mathrm{ms}$ and $8 \pm 0.02 \mathrm{MHz} / \mathrm{ms}$ at characteristic centre frequencies of 906 and $1400 \mathrm{MHz}$ respectively. Similarly, the drift rates for burst 11 were measured to be $15 \pm 0.02 \mathrm{MHz} / \mathrm{ms}, 67 \pm 0.004$ $\mathrm{MHz} / \mathrm{ms}$ and $19 \pm 0.003 \mathrm{MHz} / \mathrm{ms}$ at $906 \mathrm{MHz}, 1284 \mathrm{MHz}$ and $1400 \mathrm{MHz}$ respectively. The measured drifts rates at these frequencies have allowed us to fill in the gap between $600 \mathrm{MHz}$ and $1400 \mathrm{MHz}$ in Figure 4. The increase in magnitude of drift rate with increasing radio frequency is consistent with the results of Hessels et al. (2019). We compare our measured drift rates with the ones published for this source between $600-6500 \mathrm{MHz}$ in 

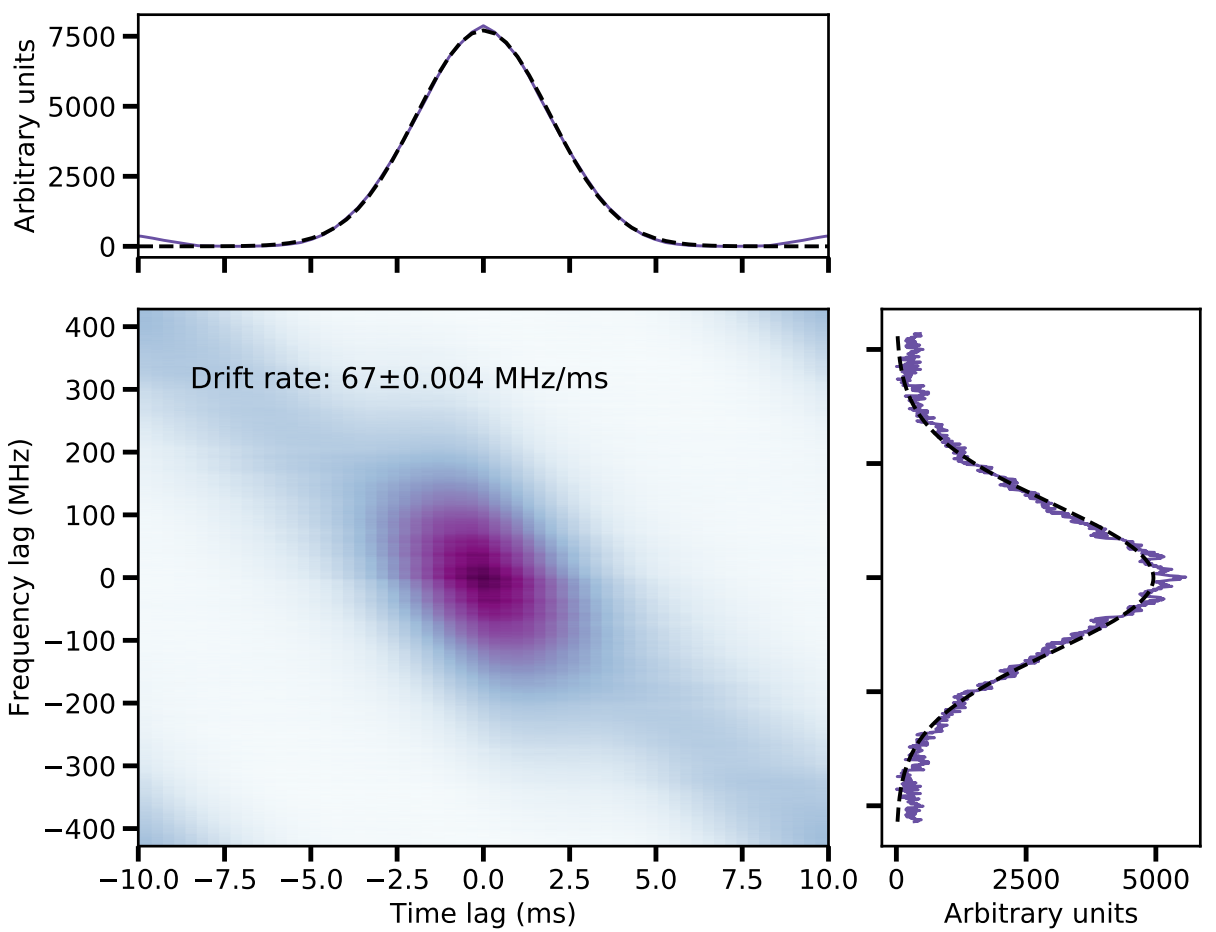

Figure 3. Autocorrelation function burst analysis for burst 11. The centre panel shows a two-dimensional ACF for the burst, with adjacent sub-panels showing the average along the time and frequency axes. These average ACF curves are fitted with Gaussian distributions.

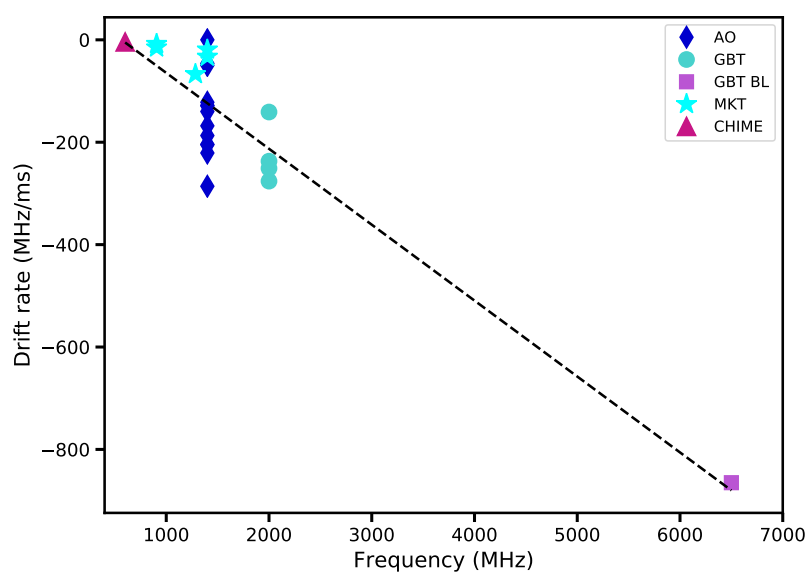

Figure 4. Measured linear burst drift rate as a function of centre frequency for the ACF method. We fit a linear drift rate evolution with slope $\alpha=$ $-0.15 \pm 0.01$.

Figure 4 and fit a linear drift rate evolution with slope $\alpha=-0.15 \pm 0.01$. We note that we do not do a weighted fit as the uncertainties are not well quantified in the literature (Hessels et al. 2019).

\subsection{Pulse periodicity}

Until recently, FRB 121102 was the only known repeating FRB and has generated much interest in searching for a possible periodicity in its pulses. The measurement of an emission period would be highly suggestive of progenitor models involving a rotating object. However, the lack of periodicity does not necessarily exclude rotating models. Two of the bursts detected by the Effelsberg radio telescope in 2017 were separated by $\sim 34 \mathrm{~ms}$ (Hardy et al. 2017). Similarly, Scholz et al. (2016) report on two bursts detected by the Green Bank Telescope and separated by $\sim 37 \mathrm{~ms}$. Figure 1 presents our real-time detections of bursts 03 and 05 on the 10th September 2019 at UTs 03:58:33.419 and 04:26:10.138 respectively. Similar to the bursts detected by the Effelsberg telescope, each of our two bursts shows a small 'precursor' separated from the main burst by $\sim 28 \mathrm{~ms}$ and $\sim 34 \mathrm{~ms}$ respectively with the signal level between the main burst and the precursor equal to the noise floor. It is apparent from Figure 1, that the precursors in bursts 03 and 05 are fainter than the main pulse. The sensitivity and large bandwidth of MeerKAT have enabled us to detect these precursors, especially in the case of burst 05 in which the precursor spans a very narrow band. Interestingly, the precursor to burst 03 also drifts towards lower frequencies and looks similar to the brighter main pulse. Periodicity searches on recorded data from 8th October 2019 yielded no significant periodic pulsations above the threshold S/N (see Section 3.1.1 for more details). The similar time scales of separation between the main pulse and precursors in all the observed cases hint towards compact emission regions. The non-detection of a periodicity does not necessarily imply a limit on any possible underlying periodicity as the source could be a rotating object in which multiple bursts were emitted during a single rotation. Recent results suggest that though there is an observed periodicity in the activity of these repeaters, intrinsic periodicity between subsequent pulses is yet to be revealed (The CHIME/FRB Collaboration et al. 2020; Rajwade et al. 2020). 


\section{CONCLUSIONS}

In this paper, we present 11 detections of single pulses from FRB 121102 during its 'active' period on 10th September 2019, using the newly commissioned MeerTRAP transient detection pipeline at the MeerKAT radio telescope in South Africa. Four of the 11 bursts $(08,09,10$ and 11) were detected simultaneously with the NRT thereby enabling us to verify and characterise our pipeline and system. Some pulses have sharp profiles while others exhibit downward drifting frequency sub-structure with increasing radio frequency. The analysis of the complex pulse structure and the structure-optimized DMs will be reported in Platts et al. (in prep). We characterize the drift rates of the pulses that exhibit sub-pulse drifting using a 2D ACF method. The measured drift rates at 906, 1284 and $1400 \mathrm{MHz}$ are found to be consistent with those published between $600-6500 \mathrm{MHz}$, with a slope of $-0.15 \pm 0.01$. Bursts 03 and 05 exhibit 'precursors' separated from the main pulse peak by $\sim 28$ and $\sim 34$ ms respectively.

A joint campaign of multi-frequency observations of FRB 121102 was carried out on 6th and 8th October 2019 between the MeerKAT (900-1670 MHz), Nançay (1100-1800 MHz) and Effelsberg (4000-8000 MHz) radio telescopes to better understand the frequency drifts and structures observed in the pulses detected in the previous observing run. The non-detections of bursts above the detection thresholds across this wide and continuous band in the radio meant that we could constrain the source to be in a truly 'inactive' state during this period. Periodicity searches were performed on recorded data but no significant periodic pulsations were detected above the threshold. The lack of a detectable period does not exclude a rotating object as a progenitor, as the detections of precursors are suggestive of compact emission regions akin to a rotating object, in which multiple bursts are emitted during a single rotation.

\section{ACKNOWLEDGEMENTS}

MC would like to thank SARAO for the approval of the MeerKAT DDT request and the CAM/CBF and operator teams for their time and effort invested in the observations. The MeerKAT telescope is operated by the South African Radio Astronomy Observatory (SARAO), which is a facility of the National Research Foundation, an agency of the Department of Science and Innovation. This work was partly based on observations with the 100-m telescope of the MPIfR (Max-Planck-Institut für Radioastronomie) at Effelsberg. The authors acknowledge funding from the European Research Council (ERC) under the European Union's Horizon 2020 research and innovation programme (grant agreement No 694745). LGS is a Lise Meitner independent research group leader and acknowledges support from the Max Planck Society. LR acknowledges the support given by the Science and Technology Facilities Council through an STFC studentship.

\section{REFERENCES}

Adámek K., Armour W., 2016, arXiv e-prints, p. arXiv:1611.09704

Adámek K., Armour W., 2019, A GPU Implementation of the Harmonic Sum Algorithm. p. 489

Adámek K., Dimoudi S., Giles M., Armour W., 2017, arXiv e-prints, p. arXiv: 1711.10855

Bassa C. G., et al., 2016, MNRAS, 456, 2196

Bolli P., et al., 2015, Journal of Astronomical Instrumentation, 4, 1550008
Caleb M., Spitler L. G., Stappers B. W., 2018, Nature Astronomy, 2, 839

Camilo F., et al., 2018, ApJ, 856, 180

Coenen T., van Leeuwen J., Hessels J. W. T., Stappers B. W., Kondratiev V. I., Alexov A., Breton R. P., Bilous 2014, A\&A, 570, A60

Desvignes G., Barott W. C., Cognard I., Lespagnol P., Theureau G., 2011. pp 349-350 (https://aip.scitation.org/doi/pdf/10.1063/1.3615154), doi:10.1063/1.3615154, https://aip.scitation.org/doi/abs/ $10.1063 / 1.3615154$

Di L., et al., 2019, The Astronomer's Telegram, 13064, 1

Dimoudi S., Armour W., 2015, arXiv e-prints, p. arXiv:1511.07343

Dimoudi S., Adamek K., Thiagaraj P., Ransom S. M., Karastergiou A., Armour W., 2018, ApJS, 239, 28

Eatough R. P., Keane E. F., Lyne A. G., 2009, MNRAS, 395, 410

Gajjar V., et al., 2018, ApJ, 863, 2

Gourdji K., Michilli D., Spitler L. G., Hessels J. W. T., Seymour A., Cordes J. M., Chatterjee S., 2019, ApJ, 877, L19

Hardy L. K., et al., 2017, MNRAS, 472, 2800

Hessels J. W. T., et al., 2019, ApJ, 876, L23

Hotan A., van Straten W., Manchester R., 2004, Publ. Astron. Soc. Australia, 21,302

Jonas J., MeerKAT Team 2016, in MeerKAT Science: On the Pathway to the SKA. p. 1

Josephy A., et al., 2019, ApJ, 882, L18

Karastergiou A., et al., 2015, MNRAS, 452, 1254

Kumar P., et al., 2019, arXiv e-prints, p. arXiv:1908.10026

Lorimer D. R., Bailes M., McLaughlin M. A., Narkevic D. J., Crawford F., 2007, Science, 318, 777

Main R., et al., 2018, Nature, 557, 522

Mauch T., et al., 2020, ApJ, 888, 61

Michilli D., et al., 2018, Nature, 553, 182

Oppermann N., et al., 2015, A\&A, 575, A118

Petroff E., et al., 2016, Publ. Astron. Soc. Australia, 33, e045

Rajwade K. M., et al., 2020, arXiv e-prints, p. arXiv:2003.03596

Rane A., Lorimer D., 2017, Journal of Astrophysics and Astronomy, 38, 55

Ransom S. M., Eikenberry S. S., Middleditch J., 2002, AJ, 124, 1788

Scholz P., et al., 2016, ApJ, 833, 177

Shannon R. M., et al., 2018, Nature, 562, 386

Spitler L. G., et al., 2014a, ApJ, 780, L3

Spitler L. G., et al., 2014b, ApJ, 790, 101

Spitler L. G., et al., 2016, Nature, 531, 202

Tendulkar S. P., et al., 2017, ApJ, 834, L7

The CHIME/FRB Collaboration et al., 2019, arXiv e-prints, p. arXiv: 1908.03507

The CHIME/FRB Collaboration et al., 2020, arXiv e-prints, p. arXiv:2001.10275

Thornton D., Stappers B., Bailes M., Barsdell B., Bates S., Bhat 2013, Science, 341, 53

Tingay S. J., et al., 2015, AJ, 150, 199

Valente G., et al., 2010, The dual-band LP feed system for the Sardinia Radio Telescope prime focus. p. 774126, doi:10.1117/12.857306

Zhang Y. G., Gajjar V., Foster G., Siemion A., Cordes J., Law C., Wang Y., 2018, ApJ, 866, 149

van Straten W., Bailes M., 2011, Publ. Astron. Soc. Australia, 28, 1

This paper has been typeset from a $\mathrm{T}_{\mathrm{E}} \mathrm{X} / \mathrm{LAT}_{\mathrm{E}} \mathrm{X}$ file prepared by the author. 